\title{
STRUCTURE THEORY AND REFLEXIVITY OF CONTRACTION OPERATORS
}

\author{
B. CHEVREAU, G. EXNER, AND C. PEARCY
}

1. Introduction. Let $\mathscr{H}$ be a separable, infinite-dimensional, complex Hilbert space, and let $\mathscr{L}(\mathscr{H})$ denote the algebra of all bounded linear operators on $\mathscr{H}$. The purpose of this note is to announce several new, and rather general, sufficient conditions that a contraction $T$ in $\mathscr{L}(\mathscr{H})$ be reflexive, and, at the same time, to give various characterizations of the class of those contractions that possess an analytic invariant subspace (definition given below). Complete proofs and other results will appear in [7]. The principal new idea involved is a considerable improvement of the main construction of $\S 3$ of [9]. The new reflexivity theorems also depend on techniques from $[\mathbf{9}, \mathbf{3}, \mathbf{1}$, and 4], and yield, in particular, the following improvement of the main result of [4].

THEOREM 1.1. If $T$ is a contraction in $\mathscr{L}(\mathscr{H})$ such that the spectrum $\sigma(T)$ of $T$ contains the unit circle $\mathbf{T}$, then either $T$ is reflexive or $T$ has a nontrivial hyperinvariant subspace.

If $T \in \mathscr{L}(\mathscr{H})$ we denote by $\mathscr{A}_{T}$ the dual algebra generated by $T$ (i.e., $\mathscr{A}_{T}$ is the smallest unital subalgebra of $\mathscr{L}(\mathscr{H})$ containing $T$ that is closed in the weak* $^{*}$ topology (which accrues to $\mathscr{L}(\mathscr{H})$ by virtue of its being the dual space of the Banach space $\mathscr{C}_{1}(\mathscr{H})$ of trace-class operators)). It follows that $\mathscr{A}_{T}$ is the dual space of $Q_{T}=\mathscr{C}_{1}(\mathscr{H}) /^{\perp} \mathscr{A}_{T}$, where ${ }^{\perp} \mathscr{A}_{T}$ is the preannihilator of $\mathscr{A}_{T}$ in $\mathscr{C}_{1}(\mathscr{H})$, under the pairing

$$
\langle A,[L]\rangle=\operatorname{tr}(A L), \quad A \in \mathscr{A}_{T}, L \in \mathscr{C}_{1}(\mathscr{H}),
$$

where $[L]$ denotes the element of the quotient space $Q_{T}$ containing the traceclass operator $L$. Thus, if $x$ and $y$ are vectors in $\mathscr{H}$, then $[x \otimes y]$ denotes the element of $Q_{T}$ containing the rank-one operator $x \otimes y$. The dual algebra $\mathscr{A}_{T}$ is said to have property $\left(\mathbf{A}_{1, \aleph_{0}}\right)$ if for any sequence $\left\{\left[L_{j}\right]\right\}_{j=1}^{\infty}$ of elements from $Q_{T}$ there exist vectors $x$ and $\left\{y_{j}\right\}_{j=1}^{\infty}$ in $\mathscr{H}$ satisfying

$$
\left[L_{j}\right]=\left[x \otimes y_{j}\right], \quad j=1,2, \ldots .
$$

If, moreover, there exists $\rho \geq 1$ (independent of the family $\left\{\left[L_{j}\right]\right\}$ ) with the property that for every $s>\rho$, the vectors $\{x\}$ and $\left\{y_{j}\right\}$ satisfying (1) can also be chosen to satisfy

$$
\|x\| \leq\left(s \sum_{k=1}^{\infty}\left\|\left[L_{k}\right]\right\|\right)^{1 / 2}, \quad\left\|y_{j}\right\| \leq\left(s\left\|\left[L_{j}\right]\right\|\right)^{1 / 2}, \quad j=1,2, \ldots,
$$

then we say that $\mathscr{A}_{T}$ has property $\left(\mathbf{A}_{1, \aleph_{0}}(\rho)\right)$.

Received by the editors September 15, 1987 and, in revised form, December 16, 1987. 1980 Mathematics Subject Classification (1985 Revision). Primary 47A15; Secondary 47A20. 
Recall that if $T$ is an absolutely continuous contraction in $\mathscr{L}(\mathscr{H})$, and $H^{\infty}(\mathbf{T})$ is the usual Hardy algebra of functions on $\mathbf{T}$, then the Sz.-NagyFoias functional calculus $\Phi_{T}: H^{\infty}(\mathrm{T}) \rightarrow \mathscr{A}_{T}$ is a weak ${ }^{*}$ continuous algebra homomorphism with range weak* dense in $\mathscr{A}_{T}$. The class $\mathbf{A}=\mathbf{A}(\mathscr{H})$ is defined to be the set of all those absolutely continuous contractions $T$ in $\mathscr{L}(\mathscr{H})$ for which $\Phi_{T}$ is an isometry; in other words, the set of such $T$ for which $\|f(T)\|=\|f\|_{\infty}$ for every $f$ in $H^{\infty}(\mathbf{T})$. Various sufficient conditions for an absolutely continuous contraction $T$ to belong to $\mathbf{A}$ are known [2]. One such is that $\sigma(T) \cap \mathbf{D}$ is dominating for $\mathbf{T}$, where $\mathbf{D}$ is the open unit disc in C. The class $\mathbf{A}_{1, \aleph_{0}}$ [resp. $\left.\mathbf{A}_{1, \aleph_{0}}(\rho)\right]$ is defined to consist of those $T$ in $\mathbf{A}(\mathscr{H})$ for which $\mathscr{A}_{T}$ has property $\left(\mathbf{A}_{1, \aleph_{0}}\right)$ [resp. $\left(\mathbf{A}_{1, \aleph_{0}}(\rho)\right]$.

2. Analytic invariant subspaces. It turns out that another concept plays a central role in the derivation of our results-namely, the notion of an analytic invariant subspace (cf. $[\mathbf{1 0}, \mathbf{3}])$. If $T$ is a contraction in $\mathscr{L}(\mathscr{H})$, $\mathscr{M} \in \operatorname{Lat}(T)$, and there exists a nonzero conjugate analytic function $e: \lambda \rightarrow e_{\lambda}$ from $\mathbf{D}$ into $\mathscr{M}$ such that

$$
(T \mid \mathscr{M}-\lambda)^{*} e_{\lambda}=0, \quad \forall \lambda \in \mathbf{D}
$$

then $\mathscr{M}$ is said to be an analytic invariant subspace for $T$. If, in addition, $\bigvee_{\lambda \in \mathrm{D}} e_{\lambda}=\mathscr{M}$, then $\mathscr{M}$ is said to be a full analytic invariant subspace for $T$.

If $T \in \mathscr{L}(\mathscr{H})$, we write $\sigma_{p}(T), \sigma_{r}(T)$, and $\sigma_{e}(T)$ for the point spectrum, right spectrum and essential (Calkin) spectrum of $T$ respectively. Moreover, following [8], we write $\mathscr{F}_{+}^{\prime}(T)$ for the set of all $\lambda$ in $\mathbf{C}$ for which $T-\lambda$ is a Fredholm operator with (strictly) positive index. Recall also that a subspace $\mathscr{K}$ of $\mathscr{H}$ is said to be semi-invariant for $T$ if $\mathscr{K}=\mathscr{M} \ominus \mathscr{N}$, where $\mathscr{M}, \mathscr{N} \in$ Lat $(T)$ and $\mathscr{M} \supset \mathscr{N}$; we denote the set of all semi-invariant subspaces for $T$ by $\mathscr{S} \mathscr{S}(T)$. (Of course, $\mathscr{H}$ itself and all elements of $\operatorname{Lat}(T)$ belong to $\mathscr{S} \mathscr{F}(T)$.) As usual, if $\mathscr{K} \in \mathscr{S} \mathscr{S}(T)$, we write $T_{\mathscr{K}}$ for the compression of $T$ to $\mathscr{K}$.

THEOREM 2.1. If $T$ is an absolutely continuous contraction in $\mathscr{L}(\mathscr{H})$, the following statements are equivalent:

(a) $T$ has an analytic invariant subspace.

(b) $T$ has a full analytic invariant subspace.

(c) $T \in \mathbf{A}_{1, \aleph_{0}}$.

(d) $T \in \mathbf{A}_{1, \aleph_{0}}(\rho)$ for some $\rho \geq 1$.

(e) There exists $\mathscr{K} \in \mathscr{S} \mathscr{F}(T)$ such that $\sigma_{p}\left(T_{\mathscr{K}}^{*}\right)=\mathbf{D}$.

(f) There exists $\mathscr{K} \in \mathscr{S} \mathscr{F}(T)$ such that $T_{\mathscr{K}} \in \mathbf{A}$ and

$$
\left(\sigma_{r}\left(T_{\mathscr{K}}\right) \cap \mathbf{D}\right) \cup\left(\mathbf{D} \backslash \mathscr{F}_{+}^{\prime}\left(T_{\mathscr{K}}\right)\right)
$$

is dominating for $\mathbf{T}$.

Some of the implications in this "wheel of equivalences" are easy; the deeper ones depend on additional, more technical, characterizations of the class $\mathbf{A}_{1, \aleph_{0}}$ in terms of certain properties $E_{\theta, \gamma}^{r}$ and $F_{\theta, \gamma}^{r}$ which appear in [9 and 7], as well as on techniques and results from $[8,4$ and 5$]$. 
3. Results on reflexivity. Recall that an operator $T$ in $\mathscr{L}(\mathscr{H})$ is said to be reflexive if every operator $S$ in $\mathscr{L}(\mathscr{H})$ such that $\operatorname{Lat}(S) \supset \operatorname{Lat}(T)$ belongs to $\mathscr{W}_{T}$, the closure of $\mathscr{A}_{T}$ in the weak operator topology. If $T$ is a contraction, we denote by $T_{a}$ the direct summand of $T$ that is the absolutely continuous part of $T$ (i.e., $T_{a}$ is the direct sum of the completely nonunitary part of $T$ and the absolutely continuous part of the unitary part of $T$ ).

THEOREM 3.1. Each of the following is a sufficient condition that an arbitrary contraction $T$ in $\mathscr{L}(\mathscr{H})$ be reflexive:

(A) $T$ (or $T^{*}$ ) satisfies any one of the conditions (a)-(f) of Theorem 2.1 .

(B) $T_{a}$ (or $\left.T_{a}^{*}\right)$ satisfies $(\mathrm{c})$ or $(\mathrm{d})$ of Theorem 2.1 .

(C) $T_{a} \in\left(C_{0} \cup C_{.0}\right) \cap \mathbf{A}$.

(D) $T_{a} \in\left(C_{1}, \cup C_{.1}\right) \cap \mathbf{A}$.

(E) $T$ is hyponormal and $T_{a} \in \mathbf{A}$.

Theorem 1.1 follows from Theorem 3.1(C) via the fact that any contraction $T$ with $\sigma(T) \supset \mathbf{T}$ not in the class $\left(C_{0} \cup C_{.0}\right) \cap \mathbf{A}$ has nontrivial hyperinvariant subspaces (cf. [2, Theorem 4.3]), and on the basis of Theorem 3.1 we make the following conjectures.

CONJECTURe 3.2 [6]. Every $T$ in $\mathbf{A}$ is reflexive.

CONJECTURE 3.3. Every hyponormal operator is reflexive.

\section{REFERENCES}

1. H. Bercovici, A contribution to the structure theory of the class A, J. Funct. Anal. 78 (1988).

2. H. Bercovici, C. Foias, and C. Pearcy, Dual algebras with applications to invariant subspaces and dilation theory, CBMS Regional Conf. Ser. in Math., no. 56, Amer. Math Soc., Providence, R.I., 1985.

3. S. Brown, Full analytic subspaces for contractions with rich spectrum, Pacific J. Math. 132 (1988), 1-10.

4. S. Brown, B. Chevreau, and C. Pearcy, On the structure of contraction operators. II, J. Funct. Anal. 76 (1988), 30-55.

5. B. Chevreau, Sur les contractions à calcul fonctionnel isométrique. I, C. R. Acad. Sci. Paris (submitted).

6. B. Chevreau, G. Exner, and C. Pearcy, Sur la reflexivité des contractions de l'espace hilbertien, C. R. Acad. Sci. Paris 305 (1987), 117-120.

7. _ On the structure of contraction operators. III, Michigan Math. J. (submitted).

8. B. Chevreau and C. Pearcy, On the structure theory of contraction operators with applications to invariant subspaces, J. Funct. Anal. 67 (1986), 360-379.

9. __ On the structure of contraction operators. I, J. Funct. Anal. 76 (1988), 1-29.

10. M. J. Cowen and R. G. Douglas, Complex geometry and operator theory, Acta Math. 141 (1978), 187-261.

U. E. R. DE Mathématiques, Université de BordeauX I, 33405 Talence, FRANCE

Department of Mathematics, Oberlin College, Oberlin, Ohio 44070

DePARTMENT OF MAThematics, UNiversity of Michigan, ANN ARbor, MICHIGAN 48109 
